\title{
Time-Frequency Analysis of Cardiac and Respiratory Parameters for the Prediction of Ventilator Weaning
}

\author{
Michele Orini, Beatriz F. Giraldo, Member, IEEE, Raquel Bailón, Montserrat Vallverdú, \\ Luca Mainardi, Salvador Benito, Ivan Díaz and Pere Caminal
}

\begin{abstract}
Mechanical ventilators are used to provide life support in patients with respiratory failure. Assessing autonomic control during the ventilator weaning provides information about physiopathological imbalances. Autonomic parameters can be derived and used to predict success in discontinuing from the mechanical support. Time-frequency analysis is used to derive cardiac and respiratory parameters, as well as their evolution in time, during ventilator weaning in 130 patients. Statistically significant differences have been observed in autonomic parameters between patients who are considered ready for spontaneous breathing and patients who are not. A classification based on respiratory frequency, heart rate and heart rate variability spectral components has been proposed and has been able to correctly classify more than $\mathbf{8 0 \%}$ of the cases.
\end{abstract}

\section{INTRODUCTION}

One of the most challenging problems in intensive care is the process of discontinuing mechanical ventilation (ventilator weaning) from patients with respiratory failure. Previous investigations reported that near $40 \%$ of the intensive care unit (ICU) patients need mechanical ventilator for sustaining their lives. Among them, $90 \%$ of the patients can be weaned from the ventilator in several days while the other 5\% $15 \%$ of the patients need longer ventilator support. However, ventilator support should be withdrawn promptly when no longer necessary so as to reduce the likelihood of known nosocomial complications and costs [1]. A failed weaning trial is discomforting for the patient and may induce significant cardiopulmonary distress. When mechanical ventilation is discontinued, up to $25 \%$ of patients have respiratory distress severe enough to necessitate reinstitution of ventilatory support [2]. Several studies have been reported on breathing pattern variability assesment [3] - [6].

Heart rate variability (HRV) is used as a noninvasive tool in the evaluation of altered autonomic nervous system

Manuscript received April 7,2008. This work was supported in part by Ministerio de Educación y Ciencia under grants TEC2007-63637 and TEC2007-68076-C02-01 from the Spanish Government.

M. Orini is with CIBER de Bioingenierá, Biomateriales y Nanomedicina (CIBER-BBN) from ISCIII, Spain, and with the Department of Bioengineering, Polytechnic University of Milan, Italy. michele@unizar.es

R. Bailón is with GTC I3A, University of Zaragoza, Spain and CIBERBNN from ISCIII

B.F. Giraldo, M. Vallverdú and P. Caminal are with Dept. of ESAII, Centre for Biomedical Engineering Research, Universitat Politècnica de Catalunya (UPC), with CIBER-BBN and with Institut de Bioenginyeria de Catalunya (IBEC), Spain. C. Pau Gargallo, 5, 08028, Barcelona, Spain. beatriz.giraldoeupc.edu

S. Benito and I. Díaz are with Emergency Department, Hospital de la Santa Creu i Sant Pau, Barcelona, Spain.

L. Mainardi is with the Department of Bioengineering, Polytechnic University of Milan, Italy
[7]. In HRV spectrum, the Low Frequency (LF) range is related to the sympathetic modulation, although its interpretation is controversial. The High Frequency (HF) range is linked to the parasympathetic activity and mainly due to respiratory sinus arrhythmia. Since ventilator weaning represents a period of transition from mechanical ventilation to spontaneous breathing and is associated with a change in autonomic activity, a change of HRV during weaning is to be expected [8]. Ventilation can alter cardiovascular function. These processes reflect the interaction between myocardial reserve, ventricular pump function, blood flow distribution, autonomic tone, lung volume and intrathoracic pressure, among others. The final response to ventilatory stress is dependent on the baseline cardiovascular state of the subject [9]. Several methods have been developed to analyze the cardiorespiratory coordination [10] - [12].

In this work, we study the cardio-respiratory interactions during discontinuation from mechanical ventilation. The goal is to assess whether differences exist in autonomic regulation between patients who are considered ready for spontaneous breathing and patients who are not. A classification based on the respiratory frequency, heart rate and spectral components of HRV, is then proposed. To estimate the time course of these cardio-respiratory parameters, their interactions and the temporal evolution of the differences between the groups, Time-Frequency analysis is used.

\section{ANALYZED DATA}

Electrocardiography (ECG) signal and respiratory flow were measured in 130 patients on weaning trials from mechanical ventilation (WEANDB data base). These patients were recorded in the Departments of Intensive Care Medicine at Santa Creu i Sant Pau Hospital and Getafe Hospital, according to a protocol approved by the local ethic committees. Using clinical criteria based on the T-tube test, the patients were classified into two groups: Group S, 91 patients with successful trials after 30 minutes, and Group F, 39 patients who failed to maintain spontaneous breathing and were reconnected after 30 minutes of weaning trial.

Respiratory flow was obtained using a pneumotachograph connected to an endotraqueal tube. The pneumotach consists of a Datex-Ohmeda monitor with a Variable-Reluctance Transducer (Validyne Model MP45-1-871). The ECG signal was obtained using a SpaceLabs Medical monitor. Both signals were recorded at a sampling frequency of $250 \mathrm{~Hz}$ during 30 minutes. The processing of the cardiac and respiratory signals obtained the $R R(k)$ and $T_{\text {Tot }}(k)$ series, containing 
the cardiac interbeat durations and the breath durations, respectively.

\section{METHODOLOGY}

\section{A. Cardio-respiratory parameters}

The ECG signal and the respiratory flow are processed in order to derive the temporal evolution of the cardiorespiratory parameters shown in Table I.

The respiratory activity of the patients is evaluated studying the respiratory frequency $f_{\mathrm{R}}(n)$ and its instantaneous variability $\breve{f}_{R}(n)$. The respiratory frequency is computed as the inverse of the series containing the breath duration, resampled at $4 \mathrm{~Hz}$. The respiratory frequency variability is derived by highpass filtering $f_{\mathrm{R}}(n)$ with a cut-off frequency of $0.03 \mathrm{~Hz}$.

In order to monitorize the cardiac activity, the heart rate $h(n)$, the HRV $\breve{h}(n)$, and the temporal evolution of its spectral components are estimated. The heart rate is derived from the QRS detection marks, following a method based on the integral pulse frequency modulation (IPFM) model, which also accounts for the presence of ectopic beats [13], using spline interpolation of order five, and a sampling rate of 4 $\mathrm{Hz}$. The HRV is obtained by highpass filtering $h(n)$ with a cut-off frequency of $0.03 \mathrm{~Hz}$.

Time-frequency analysis (TF) is performed computing the Smoothed Pseudo Wigner Ville Distribution (SPWVD) of the (analytic) HRV signal [14]. The SPWVD is a modified version of the Wigner Ville Distribution, where independent kernels are used in order to reduce the presence of the interference terms. Here, time smoothing is used with a rectangular window of 25 seconds. Exponential window with a decaying factor of $32^{-1} s^{-1}$ and frequency resolution $512^{-1} \mathrm{~Hz}$ is used for frequency smoothing.

The decomposition of the time-frequency spectra $S_{\mathrm{wv}}(f, n)$ is used to estimate the temporal evolution of LF and HF components [15]. The HF component of the HRV is known to be related with the parasympathetic system and consequently with respiration. The respiratory frequency is then used to improve the estimation of HF [16]. At every time $n$ the timefrequency spectra are divided in two bands:

$$
\begin{array}{ll}
\text { LF band: } & {\left[0.04: f_{\mathrm{R}}(n)-0.125\right] \mathrm{Hz}} \\
\text { HF band: } & {\left[f_{\mathrm{R}}(n)-0.125: f_{\mathrm{R}}(n)+0.125\right] \mathrm{Hz}}
\end{array}
$$

and the frequencies $f_{\mathrm{LF}}(n)$ and $f_{\mathrm{HF}}(n)$, and the amplitudes $A_{\mathrm{LF}}(n)$ and $A_{\mathrm{HF}}(n)$, of LF and HF are estimated as the global maxima in both frequency bands.

Normalized amplitudes $\hat{A}_{\mathrm{LF}}(n)$ and $\hat{A}_{\mathrm{HF}}(n)$, which are known to provide an estimation of the sympathovagal balance, are also taken into account [17]. Normalization consists in dividing the instantaneous amplitude of a component by the sum of both components. The difference, $e(n)$, between respiratory frequency and the frequency of the HF component of HRV is also estimated: in a normal physiological condition $f_{\mathrm{HF}}(n)$ is an expression of the respiratory sinus arrhythmia, so that a difference between $f_{\mathrm{R}}(n)$ and $f_{\mathrm{HF}}(n)$ may indicate that a sort of equilibrium is getting lost.
TABLE I

CARDIO-RESPIRATORY PARAMETERS

\begin{tabular}{cl}
\hline$f_{\mathrm{R}}(n)$ & respiratory frequency \\
$\check{f}_{\mathrm{R}}(n)$ & respiratory frequency variability \\
$h(n)$ & heart rate \\
$f_{\mathrm{LF}}(n)$ & frequency of the LF component \\
$f_{\mathrm{HF}}(n)$ & frequency of the HF component \\
$A_{\mathrm{LF}}(n)=S_{\mathrm{WV}}\left(f_{\mathrm{LF}}, n\right)$ & amplitude of the LF component \\
$A_{\mathrm{HF}}(n)=S_{\mathrm{WV}}\left(f_{\mathrm{HF}}, n\right)$ & amplitude of the HF component \\
$\hat{A}_{\mathrm{LF}}(n)=\frac{A_{\mathrm{LF}}(n)}{A_{\mathrm{LF}}(n)+A_{\mathrm{HF}}(n)}$ & normalized amplitude of the LF component \\
$\hat{A}_{\mathrm{HF}}(n)=1-\hat{A}_{\mathrm{LF}}(n)$ & normalized amplitude of the HF component \\
$e(n)=\frac{f_{\mathrm{R}}(n)-f_{\mathrm{FF}}(n)}{f_{\mathrm{R}}(n)}$ & error between $f_{\mathrm{R}}(n)$ and $f_{\mathrm{HF}}(n)$ \\
\hline
\end{tabular}

\section{B. Statistical Analysis}

The statistics shown in Table II are estimated for every cardio-respiratory parameter. The Mann Whitney test is then used to assess whether the statistics of the cardiorespiratory parameters of patients belonging to the $S$ and F groups present any significant difference. Every statistic and its corresponding $p$-value are first computed in the whole weaning process and then in a running window five minutes wide. Considering just the whole weaning trial interval, the temporal information, provided by means of time-frequency analysis, would be lost. The goal of using the running window is to estimate, for every statistical parameter, the interval where the biggest differences between the two groups appear. In this way it is possible to detect local significant differences, which may appear during just a part of the process and that would not be detected by computing the statistics for the entire duration of the trial.

Considering these parameters, stepwise multiple discriminant analysis is used to classify the patients. Stepwise method allows for the assessment of independent variables (here the statistics of the cardio-respiratory parameters) contribute significantly to the discriminant function. A first classification (DA-1) is provided using the statistical parameters computed considering the whole weaning trial. The second classification (DA-2) is provided using the parameters computed in the five minutes which, for every parameter, maximize the statistical differences between the groups. This second analysis is supposed to improve the hit ratio of the classification.

TABLE II

STATISTICS

\begin{tabular}{ll|cl}
\hline $\bar{a}$ & Mean & $a^{\sigma}$ & Statndard Deviation \\
$a^{\mathrm{m}}$ & Median & $a^{\mathrm{Iq}}$ & Interquartile Range \\
$a^{5}$ & Fifth Percentile & $a^{95}$ & Ninety-fifth Percentile \\
$a^{\mathrm{K}}$ & Kurtosis & $a^{\mathrm{Sk}}$ & Skewness \\
$a^{\mathrm{cv}}$ & Coeff of Variation & & \\
\hline
\end{tabular}




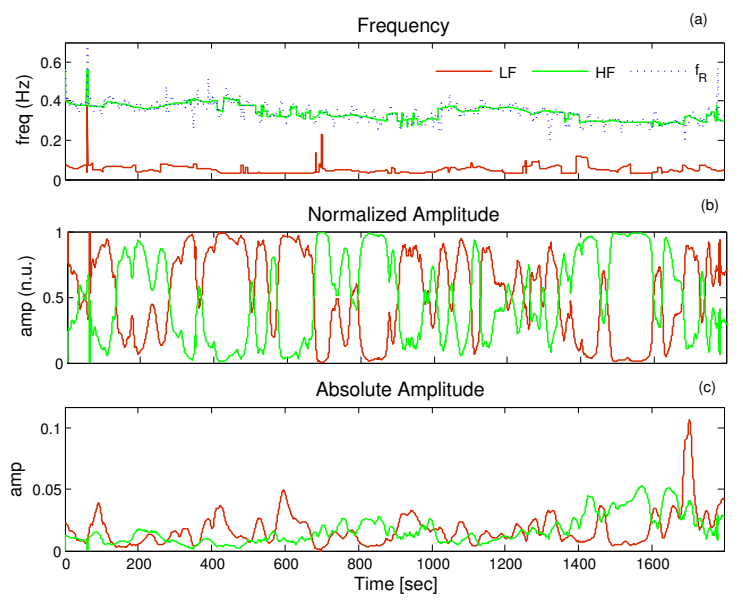

Fig. 1. Temporal evolution of the LF and HF components of HRV.

\section{Results}

For every patient the temporal evolution of 10 cardiorespiratory parameters is estimated (Table I).

As an example of decomposition of the TF spectrum, the spectral parameters of the HRV of one patient belonging to the $\mathrm{F}$ group are shown in Fig. 1. It is possible to see that $f_{\mathrm{LF}}(n)$ and $f_{\mathrm{HF}}(n)$ are quite stable, and that $f_{\mathrm{R}}(n)$ oscillates around $f_{\mathrm{HF}}(n)$ (Fig.1.a). For this patient there is not a clear prevalence of one modulation over the other one (Fig. 1.b): LF and HF components are both present and they interchange the leading role during the entire trial (the normalized amplitudes).

The central tendency and the dispersion of the parameters are summarized using 9 statistics (Table II). In Fig. 2 the median values of the respiratory frequency for all the patients are shown. The median values are computed in a window centered 8 minutes after the beginning of the trial. This is the interval which maximizes the statistical differences between the distribution of median values of groups $\mathrm{S}$ and $\mathrm{F}$. The patients who fail the weaning trial (right side) have a $f_{\mathrm{R}}^{\mathrm{m}}(n)$ significantly higher than the ones who are considered ready for the discontinuation $\left(p \approx 10^{-5}\right)$.

The differences between the two groups are assessed using the Mann Whitney Test. In Table III the $p$-values (multiplied by 1000) for every cardio-respiratory parameter and statistic are shown. These $p$-values are computed considering the whole duration of the weaning trial (about 30 minutes). Significant differences between the two groups are observed for the parameters $h(n), f_{\mathrm{R}}(n), \breve{f}_{\mathrm{R}}(n), f_{\mathrm{LF}}(n), f_{\mathrm{HF}}(n)$ and $e(n)$. The temporal evolution of the $p$-values is then obtained applying the Mann Whitney Test on the statistics iteratively computed in the running window. In Table IV the minimum $p$-values and the temporal location of the corresponding running window are shown.

In both classifications (DA-1 and DA-2), the choice of the most relevant parameters to use as independent variables in the discriminant function is performed using stepwise analysis. In the first case, three parameters are used: $f_{\mathrm{R}}^{\mathrm{m}}, \bar{h}$,

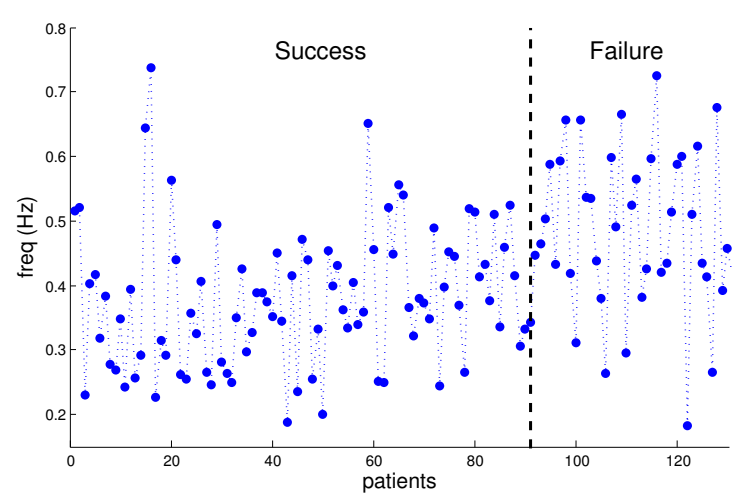

Fig. 2. $f_{\mathrm{R}}^{\mathrm{m}}(n)$ for all the patients. Patients belonging to the $\mathrm{F}$ group are observed to have higher $f_{\mathrm{R}}^{\mathrm{m}}(n)\left(p \approx 10^{-5}\right)$

$f_{\mathrm{LF}}^{\mathrm{m}}$ and $76.2 \%$ of the patients are correctly classified. In the second case, seven parameters are used as independents in the discriminant function: $f_{\mathrm{LF}}^{\mathrm{Iq}}, e^{\sigma}, h^{\mathrm{m}}, f_{\mathrm{HF}}^{\mathrm{sk}}, \bar{f}_{\mathrm{R}}, e^{\mathrm{Iq}}, A_{\mathrm{LF}}^{\mathrm{K}}$. Using these parameters about $83 \%$ of the patients are correctly classified. The classification matrix is shown in Table $V$, where the first column refers to the kind of classification (DA-1 or DA-2), S and F represent the classification as it was performed by physicians and $\hat{S}$ and $\hat{F}$ represent the predicted classification performed by discriminant analysis. All ratios refer to leave-one-out cross-validation.

TABLE III

$p$-VALUES $\left(\cdot 10^{3}\right)$ FOR THE WHOLE WEANING TRIAL

\begin{tabular}{l|lllllllll} 
& $\bar{a}$ & $a^{\sigma}$ & $a^{\mathrm{m}}$ & $a^{\mathrm{Iq}}$ & $a^{5}$ & $a^{95}$ & $a^{\mathrm{K}}$ & $a^{\mathrm{Sk}}$ & $a^{\mathrm{cv}}$ \\
\hline$h(n)$ & 3 & $\mathrm{~ns}$ & 4 & $\mathrm{~ns}$ & 2 & 18 & $\mathrm{~ns}$ & $\mathrm{~ns}$ & $\mathrm{~ns}$ \\
$f_{\mathrm{R}}(n)$ & $\dagger$ & $\mathrm{ns}$ & $\dagger$ & $\mathrm{ns}$ & 4 & $\dagger$ & $\mathrm{ns}$ & $\mathrm{ns}$ & $\mathrm{ns}$ \\
$\tilde{f}_{\mathrm{R}}(n)$ & $\mathrm{ns}$ & $\mathrm{ns}$ & $\mathrm{ns}$ & $\mathrm{ns}$ & $\mathrm{ns}$ & $\mathrm{ns}$ & $\mathrm{ns}$ & 3 & $\mathrm{~ns}$ \\
$f_{\mathrm{LF}}(n)$ & $\dagger$ & $\dagger$ & 2 & $\dagger$ & 20 & 1 & 16 & $\mathrm{~ns}$ & $\mathrm{~ns}$ \\
$f_{\mathrm{HF}}(n)$ & $\dagger$ & $\mathrm{ns}$ & $\dagger$ & $\mathrm{ns}$ & 1 & $\dagger$ & $\mathrm{ns}$ & $\mathrm{ns}$ & $\mathrm{ns}$ \\
$A_{\mathrm{LF}}(n)$ & $\mathrm{ns}$ & $\mathrm{ns}$ & $\mathrm{ns}$ & $\mathrm{ns}$ & $\mathrm{ns}$ & $\mathrm{ns}$ & $\mathrm{ns}$ & $\mathrm{ns}$ & $\mathrm{ns}$ \\
$A_{\mathrm{HF}}(n)$ & $\mathrm{ns}$ & $\mathrm{ns}$ & $\mathrm{ns}$ & $\mathrm{ns}$ & $\mathrm{ns}$ & $\mathrm{ns}$ & $\mathrm{ns}$ & $\mathrm{ns}$ & $\mathrm{ns}$ \\
$\hat{A}_{\mathrm{LF}}(n)$ & $\mathrm{ns}$ & $\mathrm{ns}$ & $\mathrm{ns}$ & $\mathrm{ns}$ & $\mathrm{ns}$ & $\mathrm{ns}$ & $\mathrm{ns}$ & $\mathrm{ns}$ & $\mathrm{ns}$ \\
$\hat{A}_{\mathrm{HF}}(n)$ & $\mathrm{ns}$ & $\mathrm{ns}$ & $\mathrm{ns}$ & $\mathrm{ns}$ & $\mathrm{ns}$ & $\mathrm{ns}$ & $\mathrm{ns}$ & $\mathrm{ns}$ & $\mathrm{ns}$ \\
$e(n)$ & $\mathrm{ns}$ & 13 & $\mathrm{~ns}$ & $\mathrm{~ns}$ & $\mathrm{~ns}$ & 43 & $\mathrm{~ns}$ & $\mathrm{~ns}$ & $\mathrm{~ns}$ \\
\hline & $\mathrm{ns}=p>0.05 ; \dagger=p<0.001$ & & & &
\end{tabular}

\section{DISCUSSION AND CONCLUSIONS}

Which criteria are the best indicators of success in discontinuation process is matter of debate. The goal of this study is to assess whether respiratory and cardiac parameters can be used as indicators of extubation readiness. The study involves 130 patients. After 30 minutes of spontaneous breathing trial, 91 patients were successfully extubated and placed in the $S$ group, while 39 were not considered ready for discontinuation and were supplied with mechanical ventilation support. They were placed in the F group. From the ECG and the respiratory flow and by means of time-frequency analysis, several cardiac and respiratory parameters are derived (Table I). Smoothed Pseudo Wigner Ville distribution was chosen for its high time-frequency resolution and for the good interference terms attenuation which it provides. The timefrequency spectra of HRV have been decomposed in order 
TABLE IV

MINIMUM $p$-VALUES $\left(\cdot 10^{3}\right)$ (ABOVE), COMPUTED IN THE RUNNING WINDOW AND ITS TEMPORAL LOCATION (BELOW).

\begin{tabular}{|c|c|c|c|c|c|c|c|c|c|}
\hline & $\bar{a}$ & $a^{\sigma}$ & $a^{\mathrm{m}}$ & $a^{\mathrm{Iq}}$ & $a^{5}$ & $a^{95}$ & $a^{\mathrm{K}}$ & $a^{\mathrm{SK}}$ & $a^{\mathrm{cv}}$ \\
\hline \multirow{2}{*}{$h(n)$} & 1 & ns & 1 & ns & $\dagger$ & 7 & ns & $\mathrm{ns}$ & ns \\
\hline & $24^{\prime}$ & & $25^{\prime}$ & & $27^{\prime}$ & $16^{\prime}$ & & & \\
\hline \multirow{2}{*}{$f_{\mathrm{R}}(n)$} & $\dagger$ & 15 & $\dagger$ & 34 & $\dagger$ & $\dagger$ & 1 & 10 & 46 \\
\hline & 7 ' & $14^{\prime}$ & $8^{\prime}$ & $12^{\prime}$ & $26^{\prime}$ & 7 & $17^{\prime}$ & $17^{\prime}$ & $26^{\prime}$ \\
\hline \multirow{2}{*}{$\check{f}_{\mathrm{R}}(n)$} & 8 & 39 & 3 & 33 & 28 & 45 & 5 & 1 & 18 \\
\hline & $21^{\prime}$ & $7^{\prime}$ & $25^{\prime}$ & $24^{\prime}$ & 24 & $14^{\prime}$ & $18^{\prime}$ & 9' & $3^{\prime}$ \\
\hline \multirow{2}{*}{$f_{\mathrm{LF}}(n)$} & $\dagger$ & $\dagger$ & $\dagger$ & $\dagger$ & 6 & $\dagger$ & 2 & 9 & 9 \\
\hline & $24^{\prime}$ & $24^{\prime}$ & $15^{\prime}$ & $16^{\prime}$ & $25^{\prime}$ & $24^{\prime}$ & $20^{\prime}$ & $20^{\prime}$ & $23^{\prime}$ \\
\hline \multirow{2}{*}{$f_{\mathrm{HF}}(n)$} & $\dagger$ & 46 & $\dagger$ & $\mathrm{ns}$ & $\dagger$ & $\dagger$ & ns & 2 & 19 \\
\hline & $8^{\prime}$ & $3^{\prime}$ & $8^{\prime}$ & & $4^{\prime}$ & $26^{\prime}$ & & 6 ' & $26^{\prime}$ \\
\hline \multirow{2}{*}{$A_{\mathrm{LF}}(n)$} & ns & ns & ns & ns & ns & 44 & 16 & 6 & 1 \\
\hline & & & & & & $25^{\prime}$ & $16^{\prime}$ & $17^{\prime}$ & $20^{\prime}$ \\
\hline \multirow{2}{*}{$A_{\mathrm{HF}}(n)$} & $\mathrm{ns}$ & $\mathrm{ns}$ & $\mathrm{ns}$ & $\mathrm{ns}$ & $\mathrm{ns}$ & $\mathrm{ns}$ & 36 & 8 & 32 \\
\hline & & & & & & & $24^{\prime}$ & $24^{\prime}$ & $26^{\prime}$ \\
\hline \multirow{2}{*}{$\hat{A}_{\mathrm{LF}}(n)$} & 27 & ns & 20 & 31 & 21 & 9 & 29 & 10 & 27 \\
\hline & $20^{\prime}$ & & 20 ' & $18^{\prime}$ & $16^{\prime}$ & $22^{\prime}$ & $18^{\prime}$ & $19^{\prime}$ & $15^{\prime}$ \\
\hline \multirow{2}{*}{$\hat{A}_{\mathrm{HF}}(n)$} & 27 & ns & 20 & 31 & 6 & 21 & 29 & 10 & 5 \\
\hline & $20^{\prime}$ & & $20^{\prime}$ & $18^{\prime}$ & $25^{\prime}$ & $16^{\prime}$ & 18 , & $19^{\prime}$ & 22 ' \\
\hline \multirow{2}{*}{$e(n)$} & ns & 1 & $\mathrm{~ns}$ & 13 & 5 & 6 & ns & 49 & 15 \\
\hline & & $26^{\prime}$ & & $12^{\prime}$ & $26^{\prime}$ & $26^{\prime}$ & & $17^{\prime}$ & $17^{\prime}$ \\
\hline
\end{tabular}

TABLE V

LEAVE-ONE-OUT VALIDATION FOR DA-1 AND DA-2

\begin{tabular}{cc|cc|c} 
& {$[\%]$} & $\mathrm{S}$ & $\mathrm{F}$ & global \\
\hline \multirow{2}{*}{ DA-1 } & $\hat{S}$ & 81.3 & 18.7 & \multirow{2}{*}{76.2} \\
& $\hat{F}$ & 35.9 & 64.1 & \\
\hline \multirow{2}{*}{ DA-2 } & $\hat{S}$ & 85.7 & 14.3 & \multirow{2}{*}{83.1} \\
& $\hat{F}$ & 23.1 & 76.9 & \\
\hline
\end{tabular}

to track the temporal evolution of LF and HF components. The respiratory frequency is used to define their frequency bands and to improve their estimation. The respiratory frequency, the heart rate and the frequency of the LF and HF components of the two groups show significant differences during the whole weaning trial. This reveals the important role of both the branches of the autonomous nervous system in the weaning success. The study of the evolution of the $p$-values suggests that not all the 30 minutes of the trial are equally useful in terms of decision for extubation. For most of the significant parameters, the $p$-value reaches its global minimum in the second part of the trial. A common feeling of anxiety among all the patients at the beginning of the weaning trial may explain the minor usefulness of the first part: it may hide the difference between the ones who are ready for spontaneous breathing and the ones who are not. The amplitude of LF and HF components are not globally relevant, unless they are normalized as $\hat{A}_{\mathrm{LF}}(n)$ and $\hat{A}_{\mathrm{HF}}(n)$. Normalized amplitudes are observed to differ in the last half of the trial, suggesting that the patients who succeed show, at that time, a prevalently sympathetic modulation.

The tracking of cardio-respiratory parameters during wean- ing shows that important differences exist in the autonomic regulation between patients who are considered ready for spontaneous breathing and patients who are not. A sequential discriminant analysis based on these cardiac and respiratory parameters has been able to correctly classify more then $80 \%$ of the patients. This work can be considered a first step toward the extraction of features which may allow automatic recognition of subjects who are ready for extubation, features which may support the right decision in discontinuation trial.

\section{ACKNOWLEDGMENTS}

The authors would like to thank to Dr. A. Ballestero and his collaborators of the Hospital Universitario de Getafe, Getafe, Spain, by his collaboration in the signal database acquisition. Thanks also to the Networking Biomedical Research Center (CIBER- BBN).

\section{REFERENCES}

[1] Jiin-Chyr Hsu, Yung-Fu Chen, Hsuan-Hung Lin, Chi-Hsiang Li and Xiaoyi Jiang, "Construction of Prediction Module for Successful Ventilator Weaning", New Trends in Applied Artificial Intelligence, 2007, pp. 766-775.

[2] M.J. Tobin, "Advances in mechanical ventilation", N. Engl. J. Med., 2001, Vol. 344, N. 26, pp. 1986-1996.

[3] M.J. Tobin, A. Jubran, F. Laghi, "Patient-Ventilator Interaction", Am J Respir Crit Care Med, , Vol. 163, pp. 1059-1063.

[4] P. Casaseca de la Higuera, M Martón-Fernández and C AlberolaLópez, "Weaning From Mechanical Ventilation: A Retrospective Analysis Leading to a Multimodal Perspective", IEEE Trans Biomed Eng, vol 53, 2006, 1330-45

[5] M.C .Khoo, "Determinants of ventilatory instability and variability", Respiration Physiology, 2000, 122(2-3), pp.167-82.

[6] Caminal P., M. Vallverdú, B. Giraldo, S. Benito, G. Vázquez, A. Voss, "Optimized symbolic dynamic approach for the analysis of the respiratory pattern", IEEE Transactions on Biomedical Engineering, 2005, vol. 52, No. 11, pp. 1832-1839.

[7] The Task Force of ESC and NASPE, "Heart rate variability. Standards of measurement, physiological interpretation, and clinical use", Eur Heart J., 1996, vol 17, 354-381

[8] Hsiu-Nien Shen, Lian-Yu Lin, Kuan-Yu Chen, Ping-Hung Kuo, Chong-Jen Yu, Huey-Dong Wu, Pan-Chyr Yang, "Changes of heart rate variability During Ventilator Weaning", Chest, 2003, 123, pp 1222-1228.

[9] M.R. Pinsky, "Cardiovascular Issues in Respiratory Care", Chest, 2005, 128 , pp. 592-597.

[10] C. Schfer, M.G. Rosenblum, J Kurths, H.H. Abel, "Heartbeat synchronized with ventilation", Nature, 1998, Vol.392, pp. 239-240.

[11] M.B. Lotric, A. Stefanovska, "Sinchronization and modulation in the human cardiorespiratory system", Phisica A., 2000, Vol.283 pp.451461.

[12] E. Toledo, S. Akselrod, I. Pinhas, D. Aravot, "Does synchronization reflect a true interaction in the cardiorespiratory system?", Med. Eng. Phys., 2002, Vol.24 pp.45-52.

[13] J. Mateo and P. Laguna,"Analysis of Heart Rate Variability in the presence of ectopic beats using the heart timing signal", IEEE Trans. Biomed. Eng., 2003, vol 50, 334-343

[14] P. Novak and V. Novak,"Time/frequency mapping of the heart rate, blood pressure and respiratory signals", Med. Biol. Eng. Comput, 1993, 31, 103-110.

[15] L. Mainardi, Montano N., And Cerutti S, "Automatic decomposition of Wigner distribution and its application to heart rate variability", Methods Inf. Med, 43; pp. 17-21, (2004)

[16] R. Bailón, P. Laguna, L. Mainardi and L. Sörnmo, "Analysis of heart rate variability using time-varying frequency bands based on respiratory frequency", Proc. of the EMBS-IEEE , 2007, 6674-6677.

[17] N. Montano, T.G. Ruscone, A. Porta, F. Lombardi, M. Pagani and A. Malliani, "Power spectrum analysis of heart rate variability to assess the changes in sympathovagal balance during graded orthostatic tilt", Circulation, 1994, vol 90, 1826-1831. 